reduces the amount of thermal energy available to the molecule to overcome its reaction barrier. But some remarkable reactions occur without the need for thermal excitation, through a process known as quantum tunnelling. Schreiner et al. ${ }^{1}$ report that just such a process occurs for $\mathrm{HCOH}$ - when the molecule adopts a certain conformation, it decays to produce formaldehyde in a matter of minutes, even though the calculated barrier for the reaction is too high to be overcome at the temperatures used in the experiments. Instead, the hydrogen atom in the hydroxyl group $(\mathrm{OH})$ of $\mathrm{HCOH}$ 'tunnels' through the barrier, in a process that relies on the wave-particle duality of the atom (Fig. 1). This unexpected reaction might explain why previous attempts to detect $\mathrm{HCOH}$ failed - the product decayed before it was found and analysed.

The authors ${ }^{1}$ investigated the quantum tunnelling process by replacing the hydrogen atom of the hydroxyl group with deuterium (a heavy isotope of hydrogen). They found that the deuterated form of $\mathrm{HCOH}$ is essentially stable under their experimental conditions. This stabilizing effect occurs because the wave associated with the deuterium atom decays before reaching the exit of the barrier (unlike the wave for the hydrogen atom), and provides direct evidence of quantum-tunnelling. Turning again to theoretical predictions to validate their results, the authors modelled the tunnelling process computationally. The half-life for $\mathrm{HCOH}$ at close to zero kelvin was predicted to be 122 minutes, whereas that of deuterated $\mathrm{HCOH}$ was predicted to be more than 1,200 years, in good agreement with their experimental observations.

The data provided by this study ${ }^{1}$ will be invaluable for those searching for $\mathrm{HCOH}$ under different conditions, for example in the gas phase, where it is expected to participate in many reactions. For such studies, it will be necessary to find out if the tunnelling process is similar in gases and solids - that is, does the matrix surrounding the $\mathrm{HCOH}$ molecules in Schreiner and colleagues' experiments have some role in the mechanism? The findings also provide a well-defined system of small molecules that will be a perfect model for studying the mechanisms of tunnelling reactions in solids. Far from being yesterday's news, small molecules still have much to teach us.

Markku Räsänen is in the Department of

Chemistry, PO Box 55, FIN-00014, University

of Helsinki, Finland.

e-mail: markku.rasanen@helsinki.fi

1. Schreiner, P. R. et al. Nature 453, 906-909 (2008)

2. Pau, C. F. \& Hehre, W. J. J. Phys. Chem. 86, 1252-1253 (1982).

3. Sodeau, J. R. \& Lee, E. K. C. Chem. Phys. Lett. 57, 71-74 (1978).

4. Baly, E. C. C., Heilbron, I. M. \& Barker, W. F. C. J. Chem. Soc Trans. 119, 1025-1035 (1921).

5. Bourissou, D., Guerret, O., Gabbai, F. P. \& Bertrand, G. Chem. Rev. 100, 39-91 (2000).

6. Whittle, E., Dows, D. A. \& Pimentel, G. C. J. Chem. Phys. 22, 1943 (1954)

\section{ANIMAL BEHAVIOUR \\ Guardian caterpillars}

Parasites are in the business of hijacking their hosts for their own purposes. A dramatic example is described by Amir Grosman and colleagues who studied behavioural changes induced in the Brazilian geometrid moth Thyrinteina leucocerae by a braconid parasitic wasp of the genus Glyptapanteles (A. H. Grosman et al. PLoS ONE 3, e2276; 2008).

The wasp lays up to 80 eggs in a moth caterpillar, where they hatch, grow and then exit to form pupae on nearby leaves three weeks later. At this point the caterpillar ceases its previous activity, and instead stands guard over the wasp pupae (pictured). While 'guarding' the pupae, caterpillars make violent swings of their heads towards any passing insect or other potential threat

Grosman et al. show that this behaviour is a direct result of parasite infestation and profits the wasp. Unlike the parasitized caterpillars, their unparasitized siblings failed to take up sentry duty when placed by wasp pupae continuing to feed as normal. Also, the head butts of parasitized caterpillars proved effective protection for the pupae, repelling nearly half of the attacks by predatory stinkbugs (Supputius cincticeps) in the laboratory. Such behaviour has no fitness advantage for the caterpillars, who die soon after the adult wasps emerge from the pupae. But the wasps profit hugely, the presence of a guarding caterpillar almost halving the mortality of pupae in field experiments.

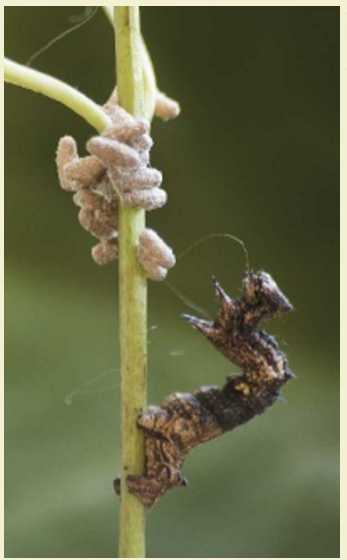

Although the mechanism by which these parasites influence their hosts is not clear, live wasp larvae were found in the bodies of caterpillars even after their broodmates had departed. Such 'brain worms' have also been seen with trematode and liver fluke parasites that modify the behaviour of their ant hosts.

Christopher Surridge

\title{
HUNTINGTON'S DISEASE
}

\section{Genetics lends a hand}

\author{
Stéphane Palfi and Bechir Jarraya
}

\section{A monkey model of Huntington's disease created by gene transfer is only a work in progress. But as a technological feat it offers great promise for fathoming this devastating condition.}

Huntington's disease is a heritable disorder that affects more than 1 in 10,000 people. Its associated neurological symptoms are severe, and there is no therapy to halt or slow its progress. To understand its pathology, and with the ultimate hope of finding a treatment, researchers have generated several experimental models of this disease ${ }^{1}$, in organisms such as flies and rodents. Although these models have led to tremendous progress in understanding the pathogenic mechanisms of Huntington's disease, none of them can replicate all the behavioural changes seen in the human disease or the changes that occur at the tissue level. The need for a primate model of the disease is thus clear.

On page 921 of this issue, Yang et al. ${ }^{2}$ describe their attempt to generate a transgenic monkey model of Huntington's*. Although only a 'proof-of-principle', their achievement is a step forward, and will undoubtedly be welcomed by those involved in developing a

${ }^{\star}$ This News \& Views article and the paper concerned ${ }^{2}$ were published online on 18 May 2008. cure for this distressing condition.

Initial symptoms of Huntington's disease, which generally appear between the ages of 30 and 40 , include changes in personality, a progressive cognitive decline and a spectrum of motor disturbances ranging from abrupt to slow and sustained involuntary movements. At a cellular level, features of the human disease include severe neuronal loss in the striatum, a deep brain structure that regulates movement and the cognitive and emotional aspects of behaviour. At the molecular level, the disease is caused by mutation in the HTT gene, which encodes the huntingtin protein. This protein normally contains a chain of 6-35 glutamine amino-acid residues. But when HTT is mutated, the number of repeats of the CAG trinucleotide, which encodes glutamine, is greatly expanded, leading to extension of the glutamine chain in huntingtin by anything from 36 to 180 repeats $^{3}$.

The technique of transgenesis is used to generate organisms carrying a specific mutation, and involves the introduction of a foreign gene into an organism. The first transgenic primate, 